Article

\title{
Upper Bounds for the Isolation Number of a Matrix over Semirings
}

\author{
LeRoy B. Beasley ${ }^{1}$ and Seok-Zun Song ${ }^{2, *(1)}$ \\ 1 Department of Mathematics and Statistics, Utah State University, Logan, UT 84322-3900, USA; \\ leroy.b.beasley@aggiemail.usu.edu \\ 2 Department of Mathematics, Jeju National University, Jeju 63243, Korea \\ * Correspondence: szsong@jejunu.ac.kr
}

Received: 5 November 2018; Accepted: 6 January 2019; Published: 9 January 2019

\begin{abstract}
Let $\mathbb{S}$ be an antinegative semiring. The rank of an $m \times n$ matrix $B$ over $\mathbb{S}$ is the minimal integer $r$ such that $B$ is a product of an $m \times r$ matrix and an $r \times n$ matrix. The isolation number of $B$ is the maximal number of nonzero entries in the matrix such that no two entries are in the same column, in the same row, and in a submatrix of $B$ of the form $\left[\begin{array}{ll}b_{i, j} & b_{i, l} \\ b_{k, j} & b_{k, l}\end{array}\right]_{\text {with nonzero entries. We know that }}$ the isolation number of $B$ is not greater than the rank of it. Thus, we investigate the upper bound of the rank of $B$ and the rank of its support for the given matrix $B$ with isolation number $h$ over antinegative semirings.
\end{abstract}

Keywords: rank; Boolean rank; isolated entry; isolation number

MSC: 15A23; 15A03; 15B34

\section{Introduction and Preliminaries}

A semiring is a set $\mathbb{S}$ with addition $(+)$ and multiplication $(\cdot)$ such that $(\mathbb{S},+)$ is an Abelian monoid with identity $0,(\mathbb{S}, \cdot)$ is a monoid with identity 1 , such that multiplication $(\cdot)$ distributes over addition (+) from both sides and such that $a \cdot 0=0 \cdot a=0$ for all $a \in \mathbb{S}$. We use juxtaposition for . for convenience. If $(\mathbb{S}, \cdot)$ is an Abelian monoid, then $\mathbb{S}$ is called a commutative semiring. If only 0 element of $\mathbb{S}$ has an additive inverse then $\mathbb{S}$ is called an antinegative semiring. Thus all rings with 1 are semirings, but no rings are antinegative. Let $\mathbb{B}=\{0,1\}$ be the binary Boolean semiring such that multiplication and addition are defined as in the integer ring except that $1+1=1$. The set, $\mathbb{Z}_{+}$, of nonnegative integers and $\mathbb{B}$, the binary Boolean semiring, are examples of antinegative semirings. Zero-divisor is defined for semirings as if $\mathbb{S}$ were a ring.

In the following, we assume that $\mathbb{S}$ is an antinegative, commutative semiring and without zero divisors and $\mathbb{B}$ is the binary Boolean semiring.

Let $\mathcal{M}_{m, n}(\mathbb{S})$ denote the set of all $m \times n$ matrices with entries in $\mathbb{S}$ with usual matrix addition and multiplication. Let $\mathcal{M}_{n}(\mathbb{S})=\mathcal{M}_{m, n}(\mathbb{S})$ if $m=n$, let $I_{m}$ denote the $m$ square identity matrix, $O_{m, n}$ denote the zero matrix in $\mathcal{M}_{m, n}(\mathbb{S})$, and $J_{m, n}$ denote the matrix of all 1's in $\mathcal{M}_{m, n}(\mathbb{S})$. If the order is obvious, then we usually omit the subscripts, and we write $I, O, J$.

The matrix $B \in \mathcal{M}_{m, n}(\mathbb{S})$ is said to be of rank $r$ if we have matrices $C \in \mathcal{M}_{m, r}(\mathbb{S})$ and $D \in \mathcal{M}_{r, n}(\mathbb{S})$ such that $B=C D$ and $r$ is the least integer with such a factorization. We denote this by $r_{\mathbb{S}}(A)=r$. Let $\mathcal{M}_{m, n}(\mathbb{B})$ denote the set of all $m \times n$ matrices with entries in $\mathbb{B}$. The Boolean rank of $D \in \mathcal{M}_{m, n}(\mathbb{B})$ is the rank over $\mathbb{B}$ and denoted $r_{\mathbb{B}}(D)$. In addition, $r_{\mathbb{S}}(O)=0$, and $O$ is the unique matrix with rank 0 .

The Boolean rank has been applied in various combinatorial theories and graph theory. As an example, if $B \in \mathcal{M}_{m, n}(\mathbb{B})$ is the adjacent matrix $G$, the bipartite graph with bipartition $(U, V)$, then the 
Boolean rank of $B$ is the least integer of bicliques that cover the edges of $G$, which is called the biclique covering number [1]. If $B \in \mathcal{M}_{m, n}(\mathbb{S})$, then the support of $B$ is the matrix $\bar{B}=\left(\overline{b_{i, j}}\right) \in \mathcal{M}_{m, n}(\mathbb{B})$ such that $\overline{b_{i, j}}=1$ if $b_{i, j} \neq 0$ and $\overline{b_{i, j}}=0$ if $b_{i, j}=0$.

We say that a matrix $B=\left[b_{i, j}\right]$ dominates a matrix $D=\left[d_{i, j}\right]$ if $b_{i, j}=0$ implies $d_{i, j}=0$.

Given a matrix $B$, we let $\mathbf{b}_{(i)}$ denote the $i^{\text {th }}$ row of $B$ and let $\mathbf{b}^{(j)}$ denote the $j^{\text {th }}$ column. Now, if $r_{\mathbb{S}}(B)=k$ and $B=C D$ is a factorization of $B \in \mathcal{M}_{m, n}(\mathbb{S})$, then $B=\mathbf{c}^{(1)} \mathbf{d}_{(1)}+\mathbf{c}^{(2)} \mathbf{d}_{(2)}+\cdots+\mathbf{c}^{(k)} \mathbf{d}_{(k)}$. Since each term $\mathbf{c}^{(i)} \mathbf{d}_{(i)}$ is a matrix of $\operatorname{rank} 1$, the rank of $B, r_{\mathbb{S}}(B)$, is the minimum number of rank 1 matrices whose sum is $B$.

Given a matrix $B \in \mathcal{M}_{m, n}(\mathbb{S})$, a set of isolated entries is a set of locations, usually written as $I=\left\{b_{i, j}\right\}$ such that $b_{i, j} \neq 0$, no two entries in $I$ are in the same column, no two entries in $I$ are in the same row, and, if $b_{i, j}, b_{k, l} \in I$, then $b_{i, l}=0$ or $b_{k, j}=0$. That is, isolated entries are independent entries and any two isolated entries $b_{i, j}$ and $b_{k, l}$ do not lie in a submatrix of $B$ of the form $\left[\begin{array}{ll}b_{i, j} & b_{i, l} \\ b_{k, j} & b_{k, l}\end{array}\right]$ with all entries nonzero. The isolation number of $B, \iota(B)$, is the maximum cardinality of a set of isolated entries. Note that $\iota(B)=0$ if and only if $B=O$.

Let $B \in \mathcal{M}_{m, n}(\mathbb{S})$ with $r_{\mathbb{S}}(B)=h$. Then, we have $h$ rank 1 matrices $B_{i}$ such that

$$
B=B_{1}+B_{2}+\cdots+B_{h}
$$

Because each matrix of rank 1 can be permuted to the form $\left[\begin{array}{ll}C & O \\ O & O\end{array}\right]$ with $\bar{C}=J$, we have that the matrix with two isolated entries of $B$ cannot be dominated by any one $B_{i}$ among the rank 1 summand of $B$ in (1). Thus,

$$
\iota(B) \leq r_{\mathbb{S}}(B)
$$

For $B=B_{1}+B_{2}+\cdots+B_{h}$, let $\mathcal{R}_{i}$ be the set of the indices of the nonzero rows of $B_{i}$ and $\mathcal{C}_{j}$ be the set of the indices of the nonzero columns of $B_{j}, i, j=1, \cdots, h$. Let $\left|\mathcal{C}_{j}\right|=c_{j}$, the number of nonzero columns of $B_{j}$ and $\left|\mathcal{R}_{i}\right|=r_{i}$, the number of nonzero rows of $B_{i}$.

Many sets, relations and functions related with matrices do not depend on the magnitude of the each entries of a matrix, but depend only on the fact of whose entry is nonzero or zero. Such combinatorially significant matrix properties have become more important in recent research. Of primary interest is the Boolean rank. Determining the rank of a Boolean matrix is known as an NP-Complete problem, (see [2]), and consequently determining upper bounds on the rank of a Boolean matrix is of importance to many authors that use Boolean rank in their research. If the Boolean matrix is the reduced adjacent matrix of some bipartite graph, the isolation number of the Boolean matrix denotes the largest size of a non-competitive matching in its bipartite graph. This fact is related to the research of such combinatorial studies as the patient hospital problem, the stable marriage problem, and so on. An additional reason for research on the set of isolation entries and isolation number is that isolation number is a lower bound on the rank of a Boolean matrix [1,3]. While determining the isolation number and determining the Boolean rank of a Boolean matrix are NP-Complete problems [4], for some matrices, determining the isolation number could be easier than determining the Boolean rank if the Boolean matrix is sparse: 
Example 1. Let

$$
B=\left[\begin{array}{lllllllll}
0 & 0 & 0 & \mathbf{1} & 0 & 1 & 1 & 1 & 1 \\
0 & 0 & 0 & 1 & \mathbf{1} & 1 & 1 & 1 & 1 \\
0 & 0 & 0 & 0 & 0 & \mathbf{1} & 1 & 1 & 1 \\
1 & 1 & 1 & 0 & 0 & 0 & 0 & 0 & 0 \\
1 & 1 & 1 & 0 & 0 & 0 & 0 & 0 & 0 \\
1 & 1 & 1 & 0 & 0 & 0 & 0 & 0 & 0 \\
\mathbf{1} & 1 & 0 & 0 & 0 & 0 & 0 & 0 & 0 \\
0 & \mathbf{1} & 1 & 0 & 0 & 0 & 0 & 0 & 0 \\
1 & 0 & \mathbf{1} & 0 & 0 & 0 & 0 & 0 & 0
\end{array}\right]
$$

The Boolean rank of $B$ is at most 6 from the first three rows and columns. However, to determine that the Boolean rank of $B$ is not 5 requires much calculation if we do not consider the isolation number. However, it is easy to see that the isolation number is 6 from the bold 1's in B, which constitute a set of isolated entries. Hence, the Boolean rank of $B$ is 6 from Equation (2).

We note that if one of the non-bold 1's were replaced by 0 , the changed matrix still has isolation number 6 and Boolean rank 6.

In this paper, we investigate the question: given a fixed $h$, if the isolation number of $B$ is $h$ what can be the upper bound of the rank of $B$ and the rank of support of $B$ ? Some terms not defined here can be found in [5] or in [6].

\section{Upper Bounds for Isolation Numbers of a Matrix in $\mathcal{M}_{m, n}(\mathbb{S})$}

Lemma 1. For $B, C \in \mathcal{M}_{m, n}(\mathbb{S})$, we have $\overline{B+C}=\bar{B}+\bar{C}$ in $\mathcal{M}_{m, n}(\mathbb{B})$.

Proof. It follows from the fact that $\mathbb{S}$ is antinegative and $1+1=1 \mathrm{in} \mathbb{B}$.

Lemma 2. For $B \in \mathcal{M}_{m, n}(\mathbb{S})$, we have $r_{\mathbb{B}}(\bar{B}) \leq r_{\mathbb{S}}(B)$.

Proof. If $r_{\mathbb{S}}(B)=h$, then $B$ has a rank-one factorization such that $B=\mathbf{c}^{(1)} \mathbf{d}_{(1)}+\mathbf{c}^{(2)} \mathbf{d}_{(2)}+\cdots+$ $\mathbf{c}^{(h)} \mathbf{d}_{(h)}$ with $C=\left[\mathbf{c}^{(1)} \mathbf{c}^{(2)} \cdots \mathbf{c}^{(h)}\right] \in \mathbf{M}_{m, h}(\mathbb{S})$ and $D=\left[\mathbf{d}_{(1)} \mathbf{d}_{(2)} \cdots \mathbf{d}_{(h)}\right]^{t} \in \mathbf{M}_{h, n}(\mathbb{S})$. Therefore, $r_{\mathbb{B}}(\bar{B})=r_{\mathbb{B}}\left(\overline{\mathbf{c}^{(1)} \mathbf{d}_{(1)}+\mathbf{c}^{(2)} \mathbf{d}_{(2)}+\cdots+\mathbf{c}^{(h)} \mathbf{d}_{(h)}}\right)=r_{\mathbb{B}}\left(\overline{\mathbf{c}^{(1)} \mathbf{d}_{(1)}}+\overline{\mathbf{c}^{(2)} \mathbf{d}_{(2)}}+\cdots+\overline{\mathbf{c}^{(h)} \mathbf{d}_{(h)}}\right) \leq h$, from Lemma 1.

Thus, $r_{\mathbb{B}}(\bar{B}) \leq r_{\mathbb{S}}(B)$.

We may have $r_{\mathbb{B}}(\bar{B})<r_{\mathbb{S}}(B)$ for some $B \in \mathcal{M}_{m, n}(\mathbb{S})$.

Example 2. Consider $A=\left[\begin{array}{ll}1 & 2 \\ 3 & 4\end{array}\right]$ and $B=\left[\begin{array}{ll}1 & 2 \\ 3 & 6\end{array}\right]$ in $\mathcal{M}_{m, n}\left(\mathbb{Z}_{+}\right)$. Then, $r_{\mathbb{Z}_{+}}(A)=2$ but $r_{\mathbb{B}}(\bar{A})=$ $r_{\mathbb{B}}\left(\left[\begin{array}{ll}1 & 1 \\ 1 & 1\end{array}\right]\right)=1$. Hence, $r_{\mathbb{B}}(\bar{A})<r_{\mathbb{Z}_{+}}(A)$. However, $r_{\mathbb{Z}_{+}}(B)=r_{\mathbb{B}}(\bar{B})=1$.

Lemma 3. For $B=\left[b_{i, j}\right] \in \mathcal{M}_{m, n}(\mathbb{S})$, we have $\iota(B)=\iota(\bar{B})$.

Proof. If $b_{i, j}$ and $b_{k, l}$ are any isolated entries in $B$, then $i \neq k$ and $j \neq l$, and that $b_{i, l}=0$ or $b_{k, j}=0$. Hence, $\overline{b_{i, j}}$ and $\overline{b_{k, l}}$ are isolated entries in $\bar{B}$, so we have $\iota(B) \leq \iota(\bar{B})$.

Conversely, if $\overline{b_{i, j}}$ and $\overline{b_{k, l}}$ are any isolated entries in $\bar{B}$, then $b_{i, j} \neq 0$ and $b_{k, l} \neq 0$ and that $b_{i, l}=\overline{b_{i, l}}=0$ or $b_{k, j}=\overline{b_{k, j}}=0$. Hence, $b_{i, j}$ and $b_{k, l}$ are isolated entries in $B$, so we have $\iota(\bar{B}) \leq \iota(B)$.

Theorem 1. If $B \in \mathcal{M}_{m, n}(\mathbb{S})$, then we have $\iota(B)=1$ if and only if $r_{\mathbb{B}}(\bar{B})=1$. 
Proof. Let $B \in \mathcal{M}_{m, n}(\mathbb{S})$. If $r_{\mathbb{B}}(\bar{B})=1$, then $B \neq O$ so that $\iota(B) \neq 0$ and since $\iota(B)=\iota(\bar{B}) \leq r_{\mathbb{B}}(\bar{B})$ by (2), we have $\iota(B)=1$.

Conversely, suppose on the contrary that there exists a matrix $B=\left[b_{i, j}\right] \in \mathcal{M}_{m, n}(\mathbb{S})$ such that $\iota(B)=1, r_{\mathbb{B}}(\bar{B})>1$. Then, there exists two non-equal and nonzero rows of $\bar{B}$, say $i$ th and $j$ th. Hence, without loss of generality, there exists a $k$ such that $\overline{b_{i, k}}=1$ and $\overline{b_{j, k}}=0$. Then, $\overline{b_{i, k}}$ and any unit entry in $j$ th row of $\bar{B}$ constitute a set of two isolated entries. Thus, $\iota(B)=\iota(\bar{B})>1$, a contradiction.

Thus, the subset of $\mathcal{M}_{m, n}(\mathbb{S})$ of matrices with isolation number 1 is the same set of matrices whose support has Boolean rank 1.

Lemma 4. Let $B \in \mathcal{M}_{m, n}(\mathbb{S})$. Then, if $r_{\mathbb{B}}(\bar{B})=2$, then $\iota(B)=2$, and if $\iota(B)=2$, then $r_{\mathbb{B}}(\bar{B}) \neq 3$.

Proof. If $r_{\mathbb{B}}(\bar{B})=2$, then we have $\iota(B)>1$ by Theorem 1 . Since $\iota(B)=\iota(\bar{B}) \leq r_{\mathbb{B}}(\bar{B})$ from Lemma 3 and (2), we have that $\iota(B)=\iota(\bar{B})=2$.

Next, suppose that $\iota(B)=2$ and $r_{\mathbb{B}}(\bar{B})=3$. Then, we have a factorization of $\bar{B}$ as $\bar{B}=C \times D$ with $C \in \mathcal{M}_{m, 3}(\mathbb{B})$ and $D \in \mathcal{M}_{3, n}(\mathbb{B})$. Then, the three rows of $D$ generate all the rows of $\bar{B}$. Since $r_{\mathbb{B}}(\bar{B})=3$, $D$ cannot have Boolean rank 2 or less. Thus, we have $r_{\mathbb{B}}(D)=3$. Therefore, we have a factorization of $D$ as $D=E \times F$ with $E \in \mathcal{M}_{3,3}(\mathbb{B})$ and $F \in \mathcal{M}_{3, n}(\mathbb{B})$. Then, the three column of $E$ generate all the columns of $D$ and $r_{\mathbb{B}}(E)=3$. Therefore, it is sufficient to consider $3 \times 3$ matrices of rank 3 . However, there are only 10 following $3 \times 3$ matrices of rank 3 up to permutations:

$$
\begin{gathered}
B_{1}=\left[\begin{array}{lll}
1 & 0 & 0 \\
0 & 1 & 0 \\
0 & 0 & 1
\end{array}\right], B_{2}=\left[\begin{array}{lll}
1 & 0 & 0 \\
0 & 1 & 0 \\
0 & 1 & 1
\end{array}\right], B_{3}=\left[\begin{array}{lll}
1 & 0 & 0 \\
0 & 1 & 0 \\
1 & 0 & 1
\end{array}\right], B_{4}=\left[\begin{array}{lll}
1 & 0 & 0 \\
0 & 1 & 0 \\
1 & 1 & 1
\end{array}\right], \\
B_{5}=\left[\begin{array}{lll}
1 & 0 & 0 \\
1 & 1 & 0 \\
0 & 0 & 1
\end{array}\right], B_{6}=\left[\begin{array}{lll}
1 & 0 & 0 \\
1 & 1 & 0 \\
0 & 1 & 1
\end{array}\right], B_{7}=\left[\begin{array}{lll}
1 & 0 & 0 \\
1 & 1 & 0 \\
1 & 0 & 1
\end{array}\right], B_{8}=\left[\begin{array}{lll}
1 & 0 & 0 \\
1 & 1 & 0 \\
1 & 1 & 1
\end{array}\right], \\
B_{9}=\left[\begin{array}{lll}
1 & 0 & 1 \\
1 & 1 & 0 \\
0 & 0 & 1
\end{array}\right], B_{10}=\left[\begin{array}{lll}
1 & 0 & 1 \\
1 & 1 & 0 \\
0 & 1 & 1
\end{array}\right] .
\end{gathered}
$$

Since $B_{5}$ can be permuted to $B_{2}$ and $B_{7}$ can be permuted to $B_{4}$, and $B_{9}$ can be permuted to $B_{6}$ with transposing. Therefore, there are only seven non-equivalent $3 \times 3$ matrices of rank 3 . However, these matrices have three isolation entries on the main diagonal. Thus, we have a contradiction to the conditions that $\iota(B)=2$ and $r_{\mathbb{B}}(\bar{B})=3$. Thus, if $\iota(B)=2$ then $r_{\mathbb{B}}(\bar{B}) \neq 3$.

Theorem 2. Let $B \in \mathcal{M}_{m, n}(\mathbb{S})$. Then, we have that $\iota(B)=2$ if and only if $r_{\mathbb{B}}(\bar{B})=2$.

Proof. From Lemma 4, we have proved the sufficiency. Thus, we will prove the necessity.

Assume that there is $A \in \mathcal{M}_{m, n}(\mathbb{S})$ such that $\iota(A)=\iota(\bar{A})=2$ and $r_{\mathbb{B}}(\bar{A})>2$. By Lemma 4, $r_{\mathbb{B}}(\bar{A}) \neq 3$, and hence $r_{\mathbb{B}}(\bar{A}) \geq 4$. Thus, we choose $B$ such that if $r_{\mathbb{B}}(\bar{B})>r_{\mathbb{B}}(\bar{C})>2$ then $\iota(C)>2$. Suppose that $\bar{B}=\overline{B_{1}}+\overline{B_{2}}+\cdots+\overline{B_{h}}$ for $h=r_{\mathbb{B}}(\bar{B})$ with $r_{\mathbb{B}}\left(\overline{B_{i}}\right)=1$. That is, $h$ is the minimum integer such that $r_{\mathbb{B}}(\bar{B})=h>2$ and $\iota(B)=2$. Suppose that the number of nonzero rows of $\overline{B_{1}}$ is the minimum among the $\overline{B_{i}}$ 's. Let us permute the rows of $\bar{B}$ so that the first $r_{1}$ rows of $\overline{B_{1}}$ are nonzero. For $j=1, \cdots, r_{1}$, let $\overline{D_{j}}$ be the matrix such that its the first $j$ row entries are the first $j$ row entries of $\bar{B}$ and whose other row entries are all zero. Let $\overline{E_{j}}$ be the matrix whose last $m-j$ row entries are the last $m-j$ row entries of $\bar{B}$ and whose other row entries are all zero. Then, $\bar{B}=\overline{D_{j}}+\overline{E_{j}}$. In addition, any set of isolated entries of $\overline{E_{j}}$ constitutes a set of isolated entries for $\bar{B}$. Now, from $r_{\mathbb{B}}(\bar{B}) \leq r_{\mathbb{B}}\left(\overline{D_{j}}\right)+r_{\mathbb{B}}\left(\overline{E_{j}}\right)$, and the fact that $r_{\mathbb{B}}\left(\overline{E_{j}}\right)=r_{\mathbb{B}}\left(\overline{E_{j-1}}\right)$ or $r_{\mathbb{B}}\left(\overline{E_{j}}\right)=r_{\mathbb{B}}\left(\overline{E_{j-1}}\right)-1$, there is some $t$ 
such that $r_{\mathbb{B}}\left(\overline{E_{t}}\right)=r_{\mathbb{B}}(\bar{B})-1$. Since $r_{\mathbb{B}}\left(\overline{E_{t}}\right)<h$ by the choice of $\bar{B}$, for this $t$, we have that $\iota\left(\overline{E_{t}}\right)>2$ since $r_{\mathbb{B}}\left(\overline{E_{t}}\right) \geq 3$. Thus, $\iota(B)=\iota(\bar{B})>2$, which is impossible since $\iota(B)=2$. Therefore, $r_{\mathbb{B}}(\bar{B})=2$.

Now, as we see in the next example, we can find some $D \in \mathcal{M}_{m, n}(\mathbb{S})$ such that $\iota(\bar{D})=3$ and $r_{\mathbb{B}}(\bar{D})>3$.

Example 3. For $n \geq 3$, let $\overline{D_{n}}=J \backslash I \in \mathcal{M}_{n}(\mathbb{B})$. Then, we can easily show that $\iota\left(\overline{D_{n}}\right)=3$ but $r_{\mathbb{B}}\left(\overline{D_{n}}\right)=h$, where $h=\min \left\{h: n \leq\left(\begin{array}{c}h \\ \left\lfloor\frac{h}{2}\right\rfloor\end{array}\right)\right\}$ [7]. Hence, $\iota\left(\overline{D_{20}}\right)=3$ but $r_{\mathbb{B}}\left(\overline{D_{20}}\right)=6$.

We define a tournament matrix $[T] \in \mathcal{M}_{n}(\mathbb{B})$ as the adjacent matrix of a directed graph that is called a tournament graph, $T$. This tournament matrix is characterized by both $[T]+[T]^{t}=J-I$ and $[T] \circ[T]^{t}=O$.

Now, we can ask the following: How much difference can there be between the isolation number of a matrix in $\mathcal{M}_{m, n}(\mathbb{S})$ and the Boolean rank of its support matrix? For each $h=1,2, \cdots, \min \{m, n\}$, can we determine the matrix forms in $\mathcal{M}_{m, n}(\mathbb{S})$ for which $\iota(A)=r_{\mathbb{B}}(\bar{A})$ ? Of course, we determined those matrices if $h=1$ or $h=2$ only in the above theorems. For $h=m$, we obtain new characterization of the matrix form in the following:

Theorem 3. Let $B \in \mathcal{M}_{m, n}(\mathbb{S})$ with $1 \leq m \leq n$. Then, $\iota(B)=r_{\mathbb{B}}(\bar{B})=m$ if and only if there are permutation matrices $P_{1} \in \mathcal{M}_{m}(\mathbb{B})$ and $P_{2} \in \mathcal{M}_{n}(\mathbb{B})$ such that $P_{1} B P_{2}=[C \mid D]$, where $\bar{C}=I_{m}+\bar{T} \in \mathcal{M}_{m}(\mathbb{B})$ and $\bar{T} \in \mathcal{M}_{m}(\mathbb{B})$ is dominated by a tournament matrix.

Proof. Suppose that $\iota(B)=m$. Then, we permute $B$ by permutation matrices $P_{1}$ and $P_{2}$ so that the set of isolated entries are in the $(b, b)$ positions, $b=1, \cdots, m$. That is, if $X=P_{1} B P_{2}$, then $I=\left\{x_{1,1}, x_{2,2}, \cdots, x_{m, m}\right\}$ is the set of isolated entries in $X$. Therefore, $X=[C \mid D]$, with $\overline{c_{i, i}}=\overline{x_{i, i}}=1$ and $\overline{c_{i, j}} \cdot \overline{c_{j, i}}=0$ for every $i$ and $j \neq i$ from the definition of the isolated entries. Thus, $\bar{C}=I_{m}+\bar{T}$ where $\bar{T}$ is an $m$-square matrix which is dominated by a tournament matrix. Thus, $P B Q=[C \mid D]$ where $\bar{C}=I_{m}+\bar{T}$ and we have no restrictions on $D$.

Conversely, if $P_{1} B P_{2}=[C \mid D]$ and $\bar{C}=I_{m}+\bar{T}$ where $\bar{T}$ is an $m$-square matrix which is dominated by a tournament matrix, then the entries on the diagonal of $C$ constitute a set of isolated entries for $P_{1} B P_{2}$. Therefore, $B$ has a set of $m$ isolated entries. Thus, $\iota(B)=r_{\mathbb{B}}(\bar{B})=m$.

Corollary 1. Let $B \in \mathcal{M}_{m, n}(\mathbb{S})$ with $1 \leq m \leq n$. If there exist permutations $P_{1} \in \mathcal{M}_{m}(\mathbb{B})$ and $P_{2} \in \mathcal{M}_{n}(\mathbb{B})$ such that $P_{1} B P_{2}=[C \mid D]$ where $C \in \mathcal{M}_{m}(\mathbb{S})$ is a diagonal matrix or a triangular matrix with nonzero diagonal entries, then $\iota(B)=r_{\mathbb{B}}(\bar{B})=m$.

\section{Conclusions}

In this paper, we investigated the upper bound of the rank of a matrix $B$ and the rank of its support for the given isolation number $k$ over antinegative semirings. Thus, we proved that the isolation number of $B$ is the same as the Boolean rank of the support of it if the isolation numbers are 1 and 2. If the isolation number were greater than 2, then we showed by example that Boolean rank of the support of the given matrix may be strictly greater than the isolation number of the matrix. In addition, in some special cases involving tournament matrices, we obtained that the isolation number of the given matrix and the Boolean rank of its support of the matrix are the same.

Author Contributions: L.B.B. and S.-Z.S. have contributed equally to this paper.

Funding: This work was supported by the research grant of Jeju National University in 2018.

Acknowledgments: The authors would like to thank to the referees for their valuable comments and suggestions on this paper.

Conflicts of Interest: The authors declare no conflict of interest. 


\section{References}

1. Gregory, D.; Pullman, N.J.; Jones, K.F.; Lundgren, J.R. Biclique coverings of regular bigraphs and minimum semiring ranks of regular matrices. J. Comb. Theory Ser. B 1991, 51, 73-89. [CrossRef]

2. Markowsky, G. Ordering D-classes and computing the Schein rank is hard. Semigr. Forum 1992, 44, 373-375. [CrossRef]

3. Beasley, L.B. Isolation number versus Boolean rank. Linear Algebra Its Appl. 2012, 436, 3469-3474. [CrossRef]

4. Akiko, K. Complexity of the sex-equal stable marriage problem (English summary). Jpn. J. Ind. Appl. Math. 1993, 10, 1-19.

5. Bondy, J.A.; Murty, U.S.R. Graph Theory; Graduate texts in Mathematics 244; Springer: New York, NY, USA, 2008.

6. Brualdi, R.; Ryser, H. Combinatorial Matrix Theory; Cambridge University Press: New York, NY, USA, 1991.

7. de Caen, D.; Gregory, D.A.; Pullman, N.J. The Boolean rank of zero-one matrices. In Proceedings of the Third Caribbean Conference on Combinatorics and Computing (Bridgetown), Univ. West Indies, Cave Hill, Barbados, 5-8 January 1981; pp. 169-173.

(c) 2019 by the authors. Licensee MDPI, Basel, Switzerland. This article is an open access article distributed under the terms and conditions of the Creative Commons Attribution (CC BY) license (http:/ / creativecommons.org/licenses/by/4.0/). 